\title{
Analyse de la Chaine de Valeurs d'oignon (Allium Cepa L.) Blanc de Soucoucoutane au Niger
}

\section{Laouali Abdoulkadri, PhD}

Département d'Agro-socio-économie, Faculté des Sciences Agronomiques, Université Boubacar Bâ de Tillaberi, Tillaberi, Niger

Assoumane Ä̈chatou, PhD

Département de Biologie, Faculté des Sciences et Techniques,

Université Abdou Moumouni, Niamey, Niger

Abdou Maman Manssour, PhD

Département Production Durable des Cultures, Faculté des Sciences

Agronomiques, Université Boubacar Bâ de Tillaberi, Tillaberi, Niger

\section{Abdoulkadri Ali,}

Département de Biologie, Faculté des Sciences et Techniques, Université Abdou Moumouni, Niamey, Niger

\section{Alzouma Mayaki Zoubeirou, Pr}

Département de Biologie, Faculté des Sciences et Techniques,

Université Abdou Moumouni, Niamey, Niger

\section{Resume}

La production d'oignon est une activité très rependue au Niger avec une diversité de variétés. Ce travail s'intéresse à l'oignon blanc «El Soucoucoutane », une variété peu connue. L'objectif de cette étude est de mettre en évidence les principaux acteurs ainsi que leurs activités à travers l'approche chaîne de valeurs. Pour évaluer les contraintes et les opportunités de cette filière oignon, la méthode SWOT a été utilisée. De ce fait, sept sites de production de cette variété d'oignon et trois marchés villageois ont été visités respectivement en avril et en mai 2018. Des entretiens individuels sur la base de choix aléatoires couplés aux focus-groups ont été conduits et ce, à différents niveaux de la chaîne. Les résultats obtenus révèlent que la chaîne de valeurs de cette variété d'oignon est courte et non structurée. Les principaux maillons sont la production, la commercialisation et la consommation. Le maillon d'approvisionnement en intrants n'est pas assez fourni. Les semences, l'engrais ainsi que les produits phytosanitaires sont pour l'essentiel autoproduits par les exploitants, véritablement orientés vers une culture biologique. Quant au maillon de la transformation, il est quasi 
inexistant avec un seul produit, le «Gabou », utilisé comme épice dans la préparation de la sauce.

Mots clés: Oignon, oignon blanc, chaîne de valeurs, Soucoucoutane, Niger

\title{
Value Chain Analysis of Soucoucoutane's White Onion (Allium cepa L.) in Niger
}

\author{
Laouali Abdoulkadri, PhD \\ Département d'Agro-socio-économie, Faculté des Sciences Agronomiques, \\ Université Boubacar Bâ de Tillaberi, Tillaberi, Niger
}

Assoumane Aichatou, PhD

Département de Biologie, Faculté des Sciences et Techniques,

Université Abdou Moumouni, Niamey, Niger

Abdou Maman Manssour, PhD

Département Production Durable des Cultures, Faculté des Sciences

Agronomiques, Université Boubacar Bâ de Tillaberi, Tillaberi, Niger

Abdoulkadri Ali,

Département de Biologie, Faculté des Sciences et Techniques,

Université Abdou Moumouni, Niamey, Niger

Alzouma Mayaki Zoubeirou, Pr

Département de Biologie, Faculté des Sciences et Techniques,

Université Abdou Moumouni, Niamey, Niger

\begin{abstract}
Onion production is a very popular activity in Niger with a diversity of varieties. This paper focuses on white onion "El Soucoucoutane", which is a little known variety. The objective of this study is to highlight the main actors and their activities across the value chain approach. To assess the constraints and opportunities of this value chain, the SWOT method was used. Thus, seven production sites of this variety of onion and three village markets were visited in April and May 2018 respectively. Individual interviews based on random choices, coupled with focus groups, were conducted at different levels of the chain. The results of the analysis revealed that the value chain of this onion variety is short and unstructured. The main links are production, marketing, and consumption. The input supply link was not sufficient. Seeds, fertilizers, and phytosanitary products are mainly self-produced by producers
\end{abstract}


whose major focus is on organic farming. As for the link of transformation, it is almost nonexistent with only one, "Gabou", used as a spice in the sauce.

Keywords: Onion, white onion, value chain, Soucoucoutane, Niger

\section{Introduction}

L'oignon est la deuxième spéculation horticole produite au monde après la tomate (D’Alessendro \& Soumah, 2008). Plus de 175 pays en produisent de par le monde (D'Alessendro \& Soumah, op . cit.) notamment le Niger. Ce pays est le deuxième producteur et principal exportateur d'oignon en Afrique de l'Ouest. Bien que les chiffres varient selon les documents, la production de cette spéculation a connu une croissance très significative au cours de ces dernières décennies. En effet, dans les années 1990, le niveau de production était d'environ 270000 tonnes ; cette production a été évaluée par l'observatoire régional de l'oignon à 447000 tonnes en 2008, mais la réalisation de 2009 a été de 384309 tonnes (PRODEX, 2013 ; RECA, 2010). L'oignon est cultivé dans toutes les régions du pays. Les bassins de production sont les plateaux de l'Ader-Doutchi, les vallées de la Maggia, et de la Tarka dans la région de Tahoua ; les vallées de Goulbi (Maradi) ; les vallées de l'Aïr (Agadez) ; la vallée de la Komadougou (Diffa) ; les Dallols Maouri et Bosso (Dosso), la Koroma (Zinder), la vallée du fleuve Niger et ses affluents (Tillabéry et Dosso). Toutefois, plus de $60 \%$ de la production nationale vient de la région de Tahoua, située au centre Sud du pays avec la variété d'oignon appelée violet de Galmi (RECA, 2010).

La filière oignon au Niger génère un chiffre d'affaires d'environ 47 milliards de F.CFA (RECA, 2011). L'exportation concerne pour l'essentiel le violet de Galmi, principale variété cultivée au Niger et la plus connue dans la sous-région (Abdou et al., 2014 ; PRODEX, 2013 ; RECA, 2011 ; RECA, 2010 ; D’Alessendro \& Suumah, 2008). Cette variété mobilise de multiples acteurs et génère des revenus importants pour les ménages tant en amont qu'en aval de la chaîne de valeurs. Outre le violet de Galmi, le Niger regorge d'autres variétés locales d'oignon (Abdou et al., 2014 ; Currah, 2002 ; Rouamba et al., 1997 ; Nabos, 1976). Parmi ces variétés d'oignon, figure la variété blanche appelée «El soucoucoutane ». C'est une variété cultivée principalement dans la commune rurale de Soucoucoutane dans le département de Dongodoutchi, région de Dosso. Cependant, cette variété reste méconnue dans la filière d'oignon du Niger.

La présente étude se propose d'une part, d'identifier et de mettre en évidence les principaux acteurs de la chaîne de valeurs de cette variété ainsi que leurs activités suivant l'approche chaîne de valeurs. D'autre part, une analyse SWOT (Strengths, Weaknesses, Opportunities and Threats) permet d'évaluer les contraintes et opportunités de cette chaîne de valeurs. 


\section{Matériel et méthode}

La démarche générale adoptée repose sur l'approche chaîne de valeurs couplée à l'analyse SWOT (Phan Dang, 2013 ; Bockel \& Tallec, 2005 ; Fabre, 1994 ; Lebailly, 1990) de la filière oignon blanc de Soucoucoutane.

\section{Zone de L'étude}

Cette étude a été conduite dans la commune rurale de Soucoucoutane située à $75 \mathrm{~km}$ au Nord de Dongondoutchi, chef-lieu de département. Comprise entre $14^{\circ} 11$ ' 12.60 " de latitude Nord et $3^{\circ} 53^{\prime}$ 6.59" de longitude Est, la commune s'étend sur une superficie de $1350 \mathrm{~km}^{2}$ (Figure 1). C'est une zone à vocation agricole caractérisée par une pluviométrie variant de faible à moyenne selon les années ; elle va de 300 à $500 \mathrm{~mm}$ de précipitation par an. La température moyenne annuelle tourne autour de $30^{\circ} \mathrm{C}$, avec un maximum de $40^{\circ} \mathrm{C}$ environ (INS, 2018). Les sols sont majoritairement sableux et peu propices à la production des cultures pluviales telles que les céréales. Cependant, ces sols sont beaucoup favorables à la production de certaines cultures maraîchères dans les localités où il y a la disponibilité de l'eau. Selon les résultats du dernier recensement général de la population, cette commune comptait 4595 habitants dont $51 \%$ de femmes (INS, 2012).

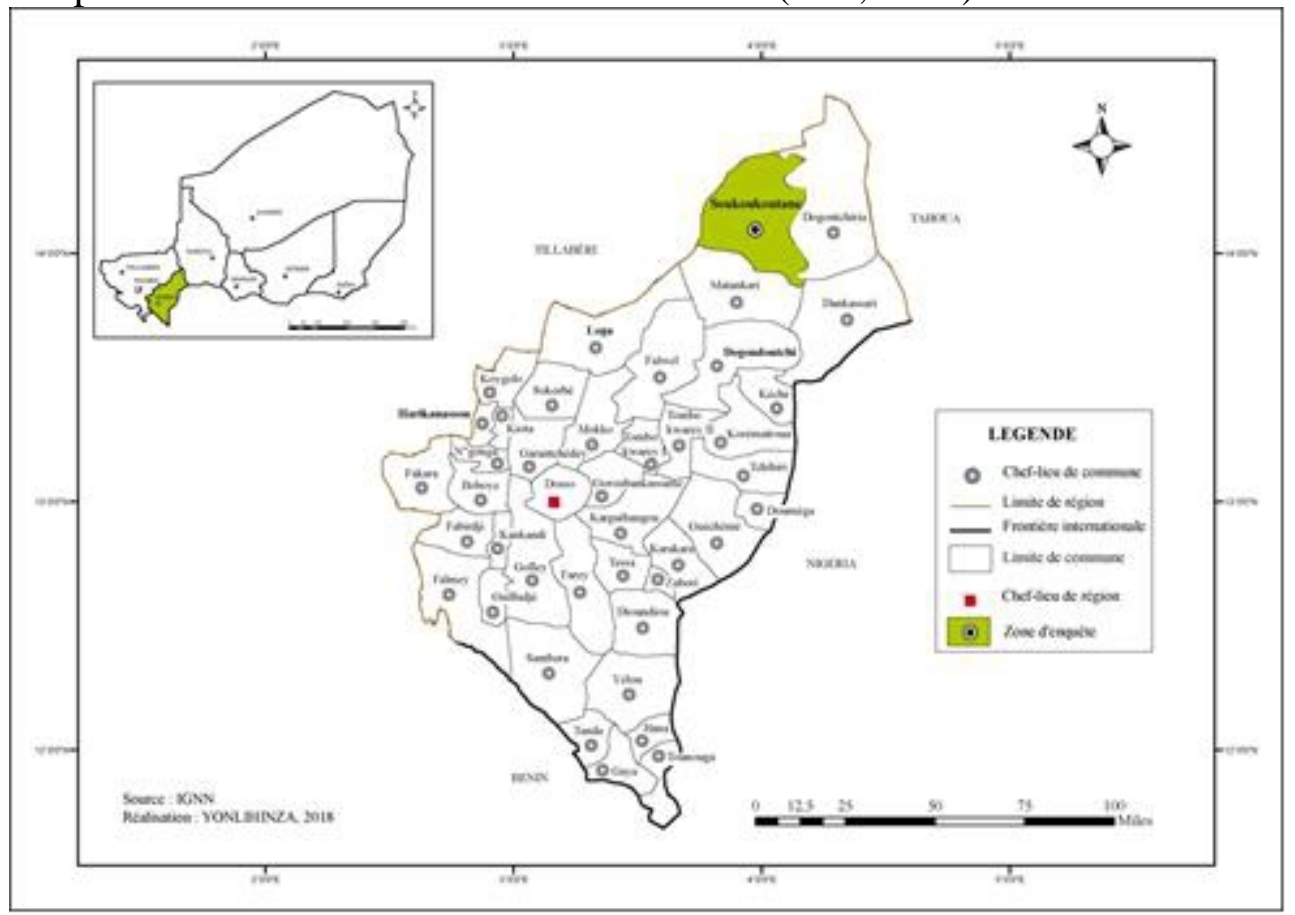

Figure 1. Localisation de la commune rurale de Soucoucoutane.

Source : Base des données Institut Géographique National du Niger (IGNN, 2018). 


\section{Echantillonnage}

La démarche générale adoptée au cours de cette étude repose sur l'approche chaîne de valeurs. Le choix d'individus à enquêter a été fait de manière aléatoire à différents niveaux de la chaîne de valeurs. Au niveau des producteurs et consommateurs, un échantillon de 30 individus a été retenu pour chacun. Pour les commerçants, un échantillon de 10 individus a été retenu sur chacun des trois marchés villageois (Bagagi, Doubalma et Kourouroubé). Quant aux producteurs de semences, quatre individus ont été aussi identifiés et enquêtés.

\section{Enquête de terrain}

L'enquête a concerné sept sites de production d'oignon blanc «El Soucoucoutane ». Il s'agit des sites de : Soucoucoutane, Kolifo, Kantcha, Dan Gari, Garin Kilbo, Gadriga Guida et Sounkourou. L'enquête a pour but de mettre en évidence les aspects agronomiques, socio-économiques et organisationnels de la chaîne de valeurs à travers le questionnement des différents maillons. Elle a consisté à :

- l'administration de questionnaire individuel auprès des producteurs, des commerçants, des fournisseurs d'intrants (multiplicateurs de semences), et des consommateurs ;

- l'organisation d'entretien en focus-group au niveau des différents sites visités ;

- l'organisation d'entretien avec les responsables communaux ; l'organisation d'entretien avec les responsables des structures paysannes.

Le travail d'enquête s'est déroulé en deux phases. La première phase a eu lieu au mois d'avril 2018. Elle été consacrée à la reconnaissance de terrain et aux entretiens, d'une part avec les autorités communales et coutumières et d'autre part, avec les responsables de structures paysannes. La deuxième phase a lieu au mois de mai 2018. Elle a été entièrement consacrée à l'administration des questionnaires individuels aux différents acteurs de la chaîne de valeurs.

\section{Saisie, Traitement et Analyse des Donnees}

Les informations collectées ont été saisies et synthétisées au moyen du tableur de traitement de données Excel. Les résultats sont présentés sous forme de tableaux et graphiques ayant fait l'objet d'analyses et commentaires. 


\section{Resultats et Discussion \\ Production \\ Profil des Producteurs}

L'analyse du Profil des producteurs indique que ces derniers sont tous de sexe masculin avec un âge moyen de 51 ans $+/-13$. La répartition par classe d'âge (Figure 2) indique que $43 \%$ ont un âge compris entre 40 et 60 ans et $30 \%$ sont âgés de plus de 60 ans. Par ailleurs, $27 \%$ des producteurs sont des jeunes âgés de moins de 40 ans.

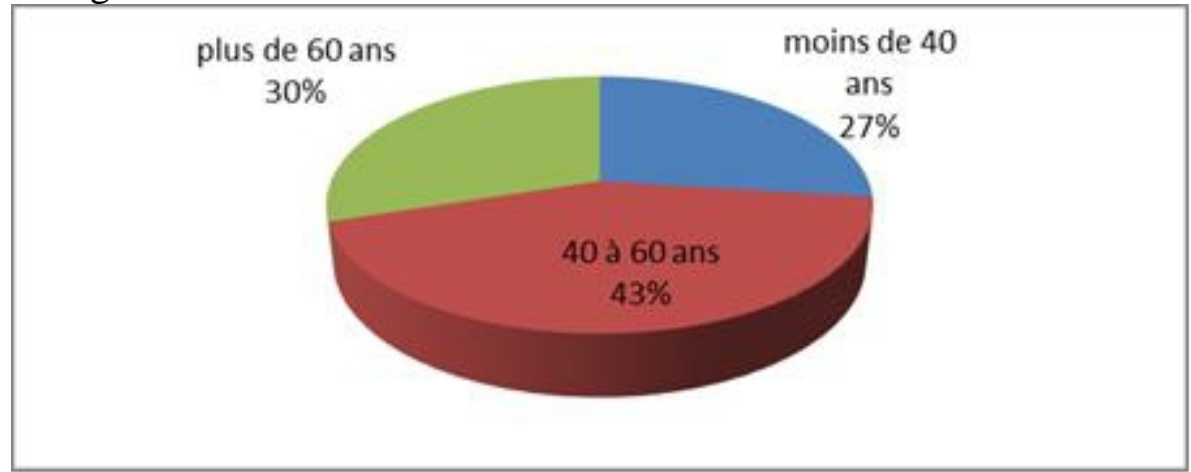

Figure 2. Répartition de l'échantillon par classe d'âge. Source : Données de l'enquête (2018).

Il ressort de l'analyse de la Figure 3 que les producteurs ont une longue expérience dans la culture de la variété étudiée, ceux qui ont moins de 5 ans d'expérience ne représentent que $27 \%$ de l'échantillon enquêté. La durée moyenne des producteurs enquêtés dans cette activité est de 16 ans. Ces producteurs ont une large tradition de la culture et de la production d'oignon à l'instar de leurs collègues situés sur les vallées Ader-Doutchi-Maggia (Tarchiani et al., 2013). Ce qui constitue un atout considérable pour la promotion de la culture de cette variété d'oignon au niveau de la zone ; la marque d'intérêt de la part des jeunes ( $27 \%$ de l'échantillon) dans cette activité est également un atout pour le développement de la filière.

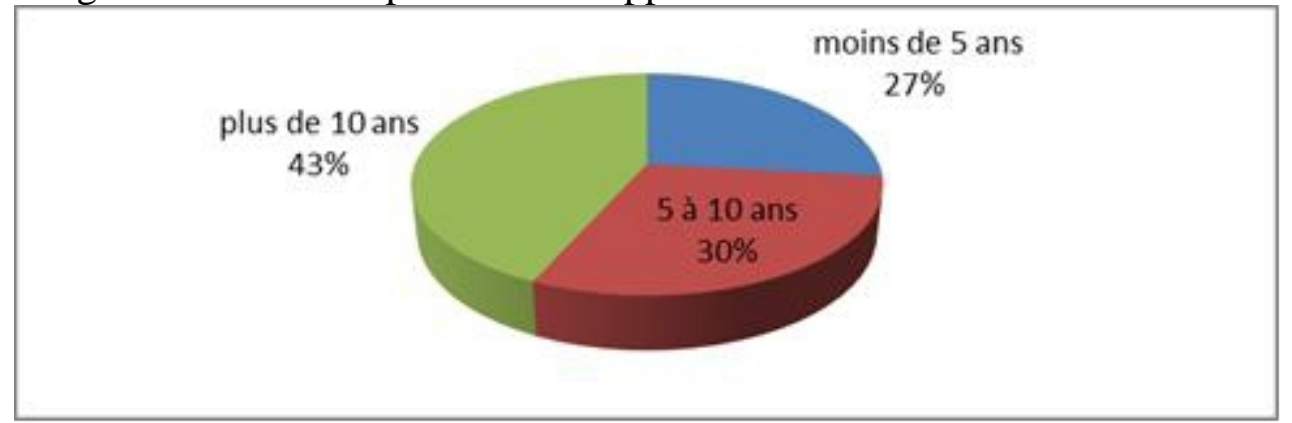

Figure 3. Répartition des individus enquêtés selon le nombre d'années d'expérience dans la production de l'oignon El Soucoucoutane.

Source : Données de l'enquête (2018). 
Au niveau organisationnel, $100 \%$ des producteurs appartiennent à des organisations paysannes. Ceci pourrait constituer un atout pour la mise à niveau de la production d'El Soucoucoutane dans la zone. Par ailleurs, il faut souligner le phénomène d'accaparement du foncier par les hommes sur les sites de production de l'oignon El Soucoucoutane caractérisé par l'absence des producteurs de sexe féminin.

\section{Approvisionnement en Intrants et Materiels Agricoles}

Le maillon de l'approvisionnement de la filière de l'oignon El Soucoucoutane se limite au système suivant : semences, engrais et produits phytosanitaires, matériel agricole. La Figure 4 présente les différentes sources d'approvisionnement des producteurs enquêtés en semences.

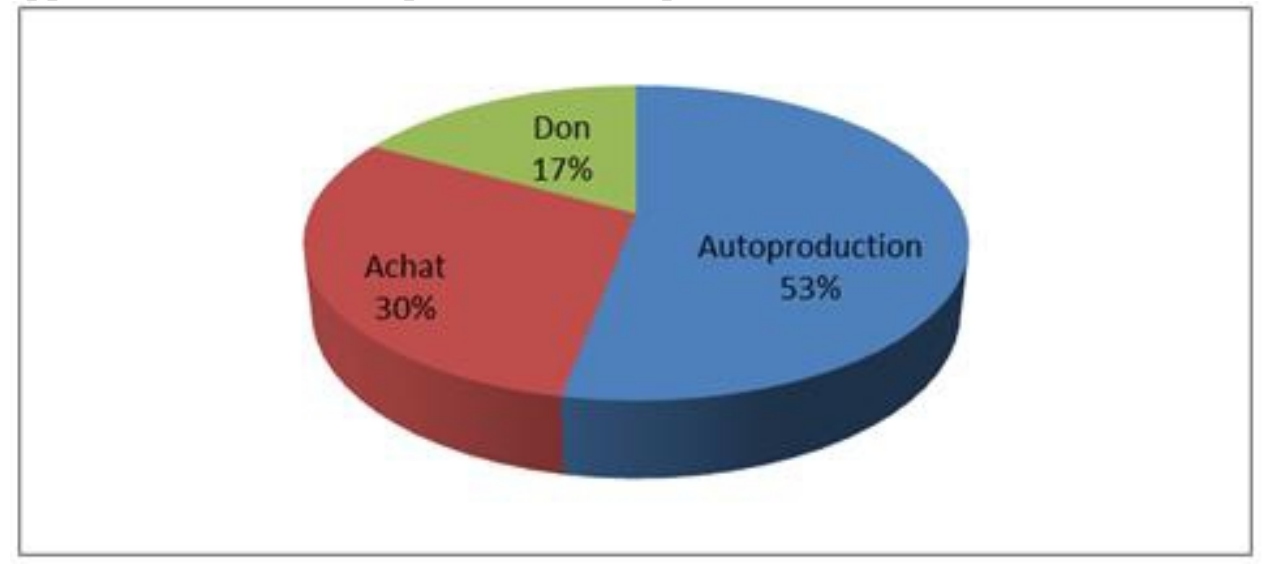

Figure 4. Différentes sources d'approvisionnement des producteurs en semences d'oignon El Soucoucoutane.

Source : Données de l'enquête (2018).

Il ressort de la Figure 4 que 53\% des producteurs enquêtés produisent eux même leur propre semence d'El Soucoucoutane. Toutefois, la proportion des producteurs qui s'approvisionnent auprès des multiplicateurs semenciers locaux (8 000 à $10000 \mathrm{~F}$ cfa la boîte de café de $100 \mathrm{~g}$ ) reste très significative et tourne autour de $30 \%$ des répondants. Les $17 \%$ affirment s'approvisionner en semence de «El Soucoucoutane » grâce au don des partenaires.

Selon les producteurs, ces semences locales semblent plus adaptées aux conditions morpho-pédologiques du milieu. Cependant, bien que ces producteurs soient engagés dans une logique de préservation et de conservation de la variété, l'étude note l'absence d'un système semencier local structuré. La Figure 5 présente la production et la conservation de semences d'oignon blanc sur le site de Soucoucoutane. 

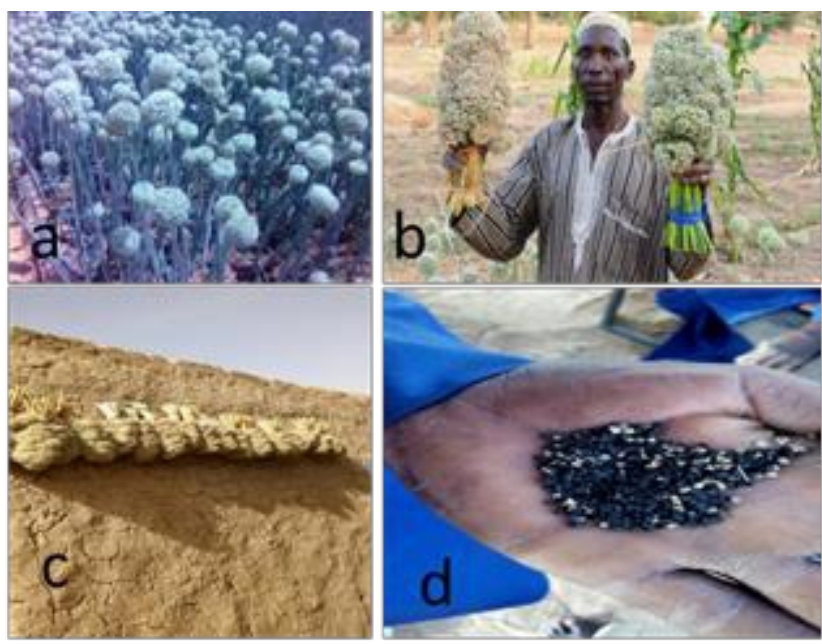

Figure 5. Production et conservation de semences d'oignon El Soucoucoutane (a. semences à maturité ; b. récoltes de semences ; c. Technique de séchage de semences ; d. semences d'oignon El Soucoucoutane).

Source : Enquête, 2018.

Par ailleurs, l'analyse du système de production d'oignon El Soucoucoutane indique que les producteurs sont orientés vers une culture biologique. L'ensemble des répondants déclarent ne pas utiliser d'engrais ni de produits phytosanitaires chimiques dans leur culture. Ils ont recours à la fumure organique issue de leur propre élevage et/ou de ceux des voisins pour fertiliser leurs parcelles. Ce choix se justifie par le souci de conservation de la production. Les répondants estiment que l'oignon produit avec de l'engrais chimique se conserve mal aussi bien en termes de durée que de la qualité du bulbe après stockage.

A cet effet, plus de $60 \%$ des répondants déclarent avoir bénéficié d'une formation en technique de compostage sous financement d'un de leurs partenaires. Toutefois, l'analyse de l'itinéraire technique de production fait ressortir une mauvaise utilisation, voire une utilisation abusive de ces engrais organiques par les producteurs. L'apport se fait presque tout le long du cycle de développement de la plante sans tenir véritablement compte du besoin réel de la plante.

Pour le Matériel agricole, il s'agit essentiellement des petits matériels aratoires très sommaires (binette, râteau, houe, pelle, arrosoir pour certains etc.). Certains sont confectionnés par les producteurs eux même à partir des objets recyclés notamment les vieux bidons de 25 litres pour l'exhaure de l'eau de l'irrigation. D'autres sont directement achetés au marché. 


\section{Superficie emblavée et rendement}

La culture d'oignon dans la commune rurale de Soucoucoutane débute à la fin de la saison de pluies (mi-septembre à fin octobre) avec le semis en pépinière. Le repiquage intervient à partir du mois de novembre sur des parcelles préalablement aménagées. La récolte a lieu trois à quatre mois au plus tard. L'analyse des superficies emblavées par les producteurs enquêtés révèle une faiblesse notoire des superficies exploitées. Elle est en moyenne de $199 \mathrm{~m}^{2}$ par exploitant en 2016-17 et $206 \mathrm{~m}^{2}$ en 2017-18 avec un rendement moyen respectif de $9,1 \mathrm{~kg} / \mathrm{m}^{2}$ et $8,8 \mathrm{~kg} / \mathrm{m}^{2}$ (Tableau 1 ).

Tableau 1. Situation globale de l'échantillon : superficie totale emblavée, production et

rendement par campagne de production

\begin{tabular}{|l|c|c|c|c|c|}
\hline Rubriques & $\begin{array}{c}\text { Superficies } \\
\text { totales }\left(\mathbf{m}^{\mathbf{2}}\right)\end{array}$ & $\begin{array}{c}\text { Productions } \\
\text { totales }(\mathbf{k g})\end{array}$ & $\begin{array}{c}\text { Rendement } \\
\mathbf{s}\left(\mathbf{k g} / \mathbf{m}^{\mathbf{2}}\right)\end{array}$ & $\begin{array}{c}\text { Superficies } \\
\text { moyennes } \\
\left(\mathbf{m}^{\mathbf{2}}\right)\end{array}$ & $\begin{array}{c}\text { Productions } \\
\text { moyennes } \\
\mathbf{( k g )}\end{array}$ \\
\hline $\begin{array}{l}\text { Campagne } \\
2016 / 17\end{array}$ & 5962 & 54098 & 9,1 & 199 & 1803 \\
\hline $\begin{array}{l}\text { Campagne } \\
2017 / 18\end{array}$ & 6168 & 54475 & 8,8 & 206 & 1816 \\
\hline
\end{tabular}

Source : Données de l'enquête (2018).

La répartition des producteurs selon la superficie emblavée confirme bien cette faiblesse des superficies exploitées. En effet, 50\% de producteurs ont moins de $200 \mathrm{~m}^{2}$ de superficie emblavée (petits producteurs); $23 \%$ ont 200 à $300 \mathrm{~m}^{2}$ d'emblavée (producteurs moyens) et seulement $27 \%$ ont plus de $300 \mathrm{~m}^{2}$ (grands producteurs en valeur relative, c'est-à-dire comparée aux valeurs des petits et moyens producteurs).

Le Tableau 2 donne la situation des campagnes 2016-17 et 2017-18 selon la catégorie de producteurs. L'analyse de ce tableau indique que les producteurs qui exploitent plus de $300 \mathrm{~m}^{2}$ de superficie présentent une situation relativement meilleure avec un rendement qui avoisine les $10 \mathrm{~kg} / \mathrm{m}^{2}$. Aussi, faudrait-il le souligner, les petits producteurs présentent des rendements intéressants comparés à la catégorie des producteurs moyens.

Le niveau de rendement des producteurs du bassin de Soucoucoutane paraît intéressant comparativement à ceux des autres bassins de production d'oignon aussi bien au Niger que dans la sous-région. Selon D'Alessendro \& Soumah (2008), avec un rendement de 34,7 tonnes/ha, le Niger présente le rendement le plus élevé de la sous-région. Pour PRODEX (2012), le rendement varie de 30 à 60 tonnes $/ \mathrm{ha}\left(3\right.$ à $\left.6 \mathrm{~kg} / \mathrm{m}^{2}\right)$ selon les variétés cultivées au Niger. Les variétés violet de Galmi et blanc de Galmi donnent respectivement un rendement de production moyen de 69,7 et 75 tonnes/ha en semis d'octobre (Assoumane \& Hilali, sd). A Niases au Sénégal, le rendement moyen de la production d'oignon tourne autour de 20 à 35 tonnes/ha (Seck, 2014). Au Burkina Faso, les rendements moyens sont de 3 à 70 tonnes/ha 
(DRARHASA, sd). Cependant, les superficies exploitées par producteur dans le cas de cette étude sont très faibles. L'INS (2012) rapporte que dans le bassin de Keita, région de Tahoua (Niger), même les petits producteurs exploitent entre 1000 et $3000 \mathrm{~m}^{2}$ de superficies.

Tableau 2. Situation des campagnes 2016-17 et 2017-18 selon la catégorie de producteurs.

\begin{tabular}{|c|c|c|c|c|}
\hline Rubriques & $\begin{array}{c}\text { Campagne } \\
\text { s } \\
\end{array}$ & $\begin{array}{c}\text { Petits } \\
\text { producteurs } \\
\end{array}$ & $\begin{array}{c}\text { Producteurs } \\
\text { moyens }\end{array}$ & $\begin{array}{c}\text { Grands } \\
\text { producteurs }\end{array}$ \\
\hline \multirow{2}{*}{$\begin{array}{l}\text { Superficie moyenne } \\
\text { emblavée }\left(\mathrm{m}^{2}\right)\end{array}$} & $2016 / 17$ & 100,0 & 238,9 & 348,8 \\
\hline & $2017 / 18$ & 88,8 & 250,1 & 385,6 \\
\hline \multirow{2}{*}{ Production moyenne $(\mathrm{kg})$} & $2016 / 17$ & 888,5 & 1931,4 & 3406,3 \\
\hline & $2017 / 18$ & 904,7 & 2033,6 & 3558,8 \\
\hline \multirow{2}{*}{ Rendement moyen $\left(\mathrm{kg} / \mathrm{m}^{2}\right)$} & $2016 / 17$ & 8,9 & 8,1 & 9,8 \\
\hline & $2017 / 18$ & 9,5 & 8,1 & 9,2 \\
\hline
\end{tabular}

Source : Données de l'enquête (2018).

\section{Contraintes à la production}

Selon les résultats de l'enquête individuelle et de focus-groups croisés avec les données des investigations de différents sites de production d'oignon El Soucoucoutane, l'eau constitue le principal facteur limitant à la production. L'irrigation se fait à partir des eaux souterraines et la nappe devient de plus en plus profonde notamment sur le site de Soucoucoutane, chef-lieu de commune. En effet, les puits, source d'alimentation en eau d'irrigation étaient à sec au passage de l'équipe au mois de mai. De l'avis des producteurs, dès fin mars le niveau des eaux de puits devenaient trop bas voire rare si bien qu'il est difficile d'assurer l'exhaure pour une bonne irrigation des planches en cette période de l'année. Ce qui n'est pas sans conséquences sur la production. Dans d'autres sites, notamment celui de Dan Gari où le niveau de la nappe est encore accessible par puisards, le problème se pose en termes de durée de vie de ces derniers et de leur capacité à assurer le besoin en eau des cultures. Aussi, le temps consacré à l'entretien presque quotidien de ces ouvrages représente-il un coût d'opportunité important pour les producteurs.

Par contre dans d'autres sites comme ceux de Kantcha et Sounkourou, le problème n'est pas celui de la disponibilité en eau, mais plutôt d'accès à la ressource faute de matériels d'irrigation adéquats (motopompe et accessoires) puisqu'ils disposent d'une mare permanente. A cela s'ajoute l'épineux problème de dégâts d'animaux sur les cultures faute de clôture appropriée (grillage).

Ces problèmes sont à la base de la faible exploitation de la surface agricole disponible. A peine $1 / 4$ de cette superficie est mis en valeur par les producteurs sur le site du village de Soucoucoutane. Aussi, faudrait-il souligner l'utilisation par les producteurs, de façon non rationnelle de l'eau caractérisée par une technique d'irrigation par gravitation, gaspilleuse de la ressource eau. 


\section{Stockage et conservation}

Les résultats de l'enquête indiquent que $50 \%$ des producteurs stockent leurs productions dans des greniers et en vrac ; $37 \%$ le font sous des hangars en vrac, $10 \%$ utilisent à la fois le grenier et le magasin pour le stockage et conservation de leurs productions d'oignon alors que seulement 3\% ont recours au magasin de conservation en matériaux définitifs pour stocker leurs productions.

La durée de conservation est très variable. Pour 57\% des répondants, elle peut aller de 3 à 6 mois voire plus si le stock est à l'abri de l'humidité et bien aéré avec un contrôle régulier. La même durée a été rapportée par PRODEX (2012). La conservation permet de différer la vente d'oignon et d'avoir un différentiel de prix significatif. En effet, à la récolte, le mana (unité de vente du produit, correspond à un sac de $65 \mathrm{~kg}$ d'oignon) se vend entre 6000 et $7000 \mathrm{~F}$ cfa dû à l'abondance du produit sur le marché. Quatre mois après, le prix du mana pourrait varier de 10000 à $12000 \mathrm{~F}$ cfa selon le marché, soit un différentiel de 4000 à 6000 Fcfa par mana. Aussi, faut-il retenir qu'aucune charge n'est à supporter pour la conservation car, les pertes liées à cela sont maitrisées par les producteurs.

\section{Commercialisation}

Le travail d'enquête a été réalisé au niveau de trois marchés et a concerné neuf commerçants qui sont tous des détaillants. Il s’agit du Marché de Bagagi avec six commerçants, celui de Doubalme et de Kourouroubé avec respectivement un (01) et deux (02) commerçants. L'enquête n'a pas pu identifier des commerçants grossistes dans le circuit de commercialisation d'oignon El Soucoucoutane dans la zone de production et son voisinage. Cela pourrait s'expliquer par le caractère très endogène de la production d'une part, et le niveau de production trop faible pour intéresser les grossistes d'autre part.

De l'analyse des résultats de l'enquête, il ressort que près de $95 \%$ de la production sont mises sur le marché par les producteurs. Le reste est affecté à l'autoconsommation, à la rémunération en nature de la main d'œuvre et autres dons. La commercialisation se fait soit par les producteurs eux-mêmes qui se transforment en commerçants détaillants et font le tour des marchés de la zone ; soit par des commerçants détaillants des légumes qui s'approvisionnent directement auprès des producteurs qui viennent écouler leurs produits sur le marché.

\section{Ecoulement des produits par les producteurs}

L'essentiel de la production est vendue aussitôt après la récolte en mars et avril. Selon l'enquête, près de $40 \%$ des producteurs vendent leur production dès la récolte afin de pouvoir répondre aux besoins de la famille, principalement sur le plan alimentaire. La situation est beaucoup plus 
frappante ces dernières années car les campagnes agricoles ont été très déficitaires dans la zone. La commercialisation se fait individuellement. Les résultats de 1'enquête révèlent que $13 \%$ des producteurs enquêtés ont vendu leurs productions sur le site ; $7 \%$ à domicile ; $80 \%$ ont écoulé leurs produits soit sur le marché de leur village et/ou sur d'autres marchés de la zone notamment à Doubalma, Kourouroube, Ayawa, d'autres jusqu'à Sanam.

A la récolte au mois de mars, le sac de $65 \mathrm{~kg}$ se vendait entre 6000 et $7000 \mathrm{~F}$ cfa sur le site ou $8000 \mathrm{~F}$ cfa au marché. Ce prix peut aller jusqu'à 10 $000 \mathrm{~F}$ cfa au mois de mai voire $15000 \mathrm{~F}$ cfa en septembre si le produit est bien conservé. Généralement, la vente se fait sans intermédiaire entre le producteur et l'acheteur (circuit court : $77 \%$ des répondants). Seulement $23 \%$ sollicitent le service d'intermédiaire pour écouler leurs productions.

\section{Ecoulement par les commerçants}

Dans la zone de l'étude, la commercialisation d'oignon, toutes variétés confondues, est exercée essentiellement par des hommes. On y rencontre aussi bien des nationaux que des commerçants détaillants venus du Nigeria voisin.

$\mathrm{Au}$ passage de l'équipe, deux variétés d'oignons (blanc de Soucoucoutane et le violet) ont été recensées sur les marchés visités à l'exception de celui de Bagagi où le blanc de Soucoucoutane est absent. Sur ce marché, l'oignon El Soucoucoutane est très peu connu. Le commerce d'oignon est détenu par les commerçants nigérians. Ces derniers s'approvisionnent directement chez eux au Nigeria et ne vendent que le violet de Galmi dont l'approvisionnement se fait tout le long de l'année.

Les commerçant détaillants nigériens s'approvisionnent régulièrement à partir de mi-mars jusqu'à fin avril auprès des producteurs soit sur le site de production ou sur les différents marchés hebdomadaires de la zone de production d'oignon El Soucoucoutane. Ils achètent le sac entre $6000-8000$ $\mathrm{F}$ cfa (à la récolte) pour le revendre en détail à 9000 voire $10000 \mathrm{~F}$ cfa. Mais faute de moyens financiers et/ou de conservation, le stock de ces commerçants en ce produit dépasse rarement trois sacs, juste de quoi faire le tour des marchés hebdomadaires de la zone jusqu'à l'épuisement de stocks de producteurs. Le même niveau de prix a été relevé par la Chambre régionale d'Agriculture de Dosso et la Fédération des coopératives maraîchères du Niger (CRA \& FCMN, 2018).

Il faut souligner que de l'avis des personnes rencontrées, le principal centre d'écoulement de l'oignon blanc El Soucoucoutane en dehors du bassin de production, c'est le marché de Sanam. C'est un marché qui présente des marges bénéficiaires intéressantes pour les producteurs. Sur ce marché, le sac de $65 \mathrm{~kg}$ d'oignon se vend entre 12000 et $13000 \mathrm{~F}$ cfa contre $8000 \mathrm{~F}$ cfa sur le marché local. Mais l'absence de route praticable limite l'accès à ce marché. A cela s'ajoute le coût élevé du transport de la marchandise (1 $500 \mathrm{~F}$ cfa par 
mana) et le problème d'insécurité qui prévaut à Sanam sur la frontière avec le Mali.

\section{Déterminants du prix de vente}

Selon les données de l'enquête, $50 \%$ des producteurs et $100 \%$ des commerçants enquêtés estiment que le prix de vente d'oignon est fonction de la variété. L'oignon El Soucoucoutane est plus cher que le violet de Galmi. Pour $17 \%$ des répondants, c'est plutôt la qualité tandis que 13\% d'autres pensent que le prix est fonction à la fois de l'abondance de la production et de la période de vente. Seulement $7 \%$ estiment que le prix est fonction du lieu d'écoulement du produit.

\section{Contraintes à la commercialisation \\ ○ Pour les producteurs}

L'analyse des données de l'enquête indique que la principale contrainte à la commercialisation de l'oignon El Soucoucoutane est le manque de débouchés. Elle a été évoquée par $72 \%$ des répondants. Puis vient la faiblesse de prix évoquée par $28 \%$ des répondants. La concurrence d'autres variétés d'oignon n'a été indiquée que par $10 \%$ des répondants. Dans le même temps, $10 \%$ d'enquêtés déclarent ne rencontrer aucune contrainte à la vente de leur produit. En effet, selon les déclarations des répondants, l'oignon El Soucoucoutane est un produit très spécifique avec des vertus et des qualités qui le distinguent d'autres variétés d'oignon et reconnus par les clients.

- Pour les commerçants détaillants

Les principales contraintes évoquées par ces commerçants sont :

- l'insuffisance de la production pour couvrir les besoins des clients,

- la disponibilité très saisonnière de l'oignon El Soucoucoutane,

- la concurrence de certains producteurs devenus commerçants détaillants opportunistes.

De manière générale, la commercialisation d'oignon El Soucoucoutane reste très informelle, individuelle et non structurée contrairement à la variété violet de Galmi (CNRA, 2013 ; Van Vugt et al., 2010).

\section{Transformation}

$\mathrm{Au}$ niveau de la zone d'étude, la transformation de l'oignon El Soucoucoutane se limite exclusivement au produit appelé «Gabou ». Cette activité est du ressort des femmes et reste très marginale. Il existe deux types de gabou :

- Le gabou préparé à base des feuilles d'oignon. Ces dernières sont généralement transformées en boulettes et utilisées comme condiment dans la préparation de la sauce (Figure 6); 
- Le gabou préparé à partir des bulbes d'oignon. Pour sa préparation, les femmes utilisent, très souvent les bulbes dont la qualité n'est pas favorable à la conservation et/ou celles qui ont entamé le processus de décomposition. De l'avis des certaines femmes enquêtées, les hommes préfèrent vendre leurs oignons en l'état que de les transformer sous forme de gabou. Ce produit est utilisé comme épice dans la sauce.
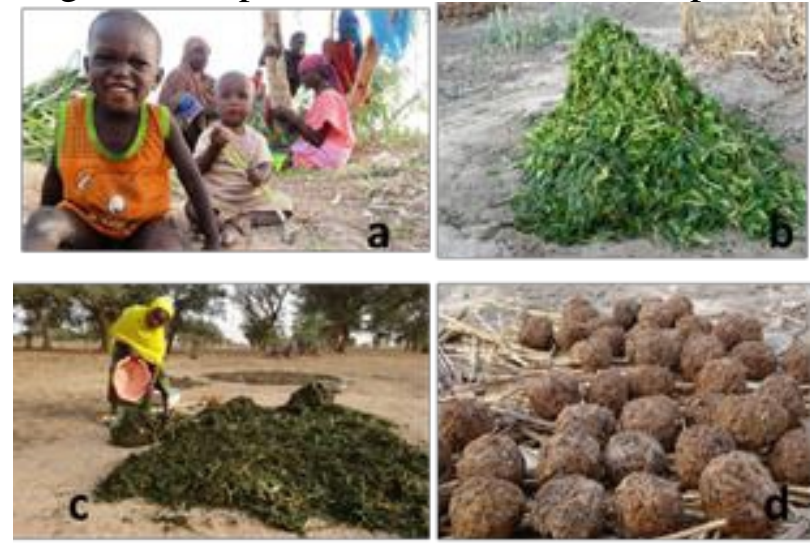

Figure 6. Préparation de Gabou à base de feuilles ( $a, b$ et $\mathrm{c}$ ) et bulbes (d) d'oignon.

Source : Enquête, 2018.

\section{Consommation}

Selon les résultats de l'enquête, les variétés d'oignon connues par les consommateurs sont le violet de Galmi et le blanc de Soucoucoutane. Et 85\% des répondants déclarent préférer le blanc de Soucoucoutane au violet de Galmi. Parmi eux $71 \%$ le préfèrent pour son goût sucré et moins piquant et sa dissolution facile dans la sauce, et $19 \%$ pour question d'habitude. En effet, selon les consommateurs, le blanc de Soucoucoutane se consomme de différentes manières (cru, bouilli, grillé, dans de la salade, dans la sauce etc.). Aussi, $76 \%$ des consommateurs enquêtés estiment que le blanc de Soucoucoutane se conserve mieux que le violet de Galmi compte tenu de la qualité du bulbe (plus ferme et change facilement d'épiderme au fur et à mesure de sa conservation).

Par ailleurs, il ressort de l'enquête et des différents entretiens en focusgroup que l'oignon El Soucoucoutane est recherché par les consommateurs pour ses diverses vertus :

- $\quad$ Contre le coût de chaleur ;

- Contre les vers intestinaux ;

- Améliore la vision ;

- Traite la fièvre aussi bien chez les humains que chez les animaux ;

- Traite les problèmes urinaires ;

- Remède contre l'angine ; 
- $\quad$ Antidote contre les morsures de serpent et les piqures de scorpion ;

- Etc.

Dans le cadre de l'analyse prospective des produits issus de la transformation d'oignon El Soucoucoutane et de l'analyse des souhaits des consommateurs, l'étude s'est intéressée à la fabrication et la mise sur le marché de nouveaux produits à forte valeur. Ainsi, il ressort que les consommateurs n'ont aucune connaissance de sous-produit à base d'oignon en dehors de Gabou.

\section{Analyse économique}

L'analyse des comptes d'exploitation des producteurs fait ressortir des différences selon les catégories des producteurs.

La charge d'exploitation est de $345 \mathrm{~F} \mathrm{cfa} / \mathrm{m}^{2}$ pour les petits producteurs (moins de $200 \mathrm{~m}^{2}$ ) contre en moyenne $450 \mathrm{~F}$ pour les deux autres catégories des producteurs. Le revenu tiré par les producteurs exploitants moins de 200 $\mathrm{m}^{2}$ est en moyenne de l'ordre de $579 \mathrm{~F}$ cfa $/ \mathrm{m}^{2}$ (Tableau 3 ). Ce revenu est de $1078 \mathrm{~F} \mathrm{cfa} / \mathrm{m}^{2}$ pour les producteurs dont les superficies emblavées sont comprises entre 200 et $300 \mathrm{~m}^{2}$. Il atteint les $1575 \mathrm{~F} \mathrm{cfa} / \mathrm{m}^{2}$ pour les producteurs exploitant plus de $300 \mathrm{~m}^{2}$.

Tableau 3. Exemple d'un compte d'exploitation de producteurs exploitant moins de $200 \mathrm{~m}^{2}$ de superficie

\begin{tabular}{|c|r|c|r|}
\hline Charges & Montants (F cfa) & Produits & Montants (F cfa) \\
\hline Achat Semences et/ou pépinière & 24000 & Production & 136000 \\
\hline Fumure organique & 20000 & Semences produites & 24000 \\
\hline Main d'œuvre & 21000 & Total 2 & 171000 \\
\hline Coût de transport & 4000 & \\
\hline Total 1 & 69000 & \\
\hline Solde & 102000 & \\
\hline Revenu/m2 & \multicolumn{3}{|c|}{$579 \mathrm{~F} \mathrm{cfa}$} \\
\hline
\end{tabular}

Ainsi, les grands producteurs enregistrent des résultats d'exploitation plus performants. Cependant, les charges des producteurs d'oignon El Soucoucoutane, toutes catégories confondues, sont nettement supérieures à celles des producteurs d'oignon dans la zone de Gotheye $\left(190 \mathrm{~F} \mathrm{cfa} / \mathrm{m}^{2}\right)$ et celle d'Ayorou ( $63 \mathrm{~F} \mathrm{cfa} / \mathrm{m}^{2}$ ) dans la région de Tillabéri au Niger (Maman Moustapha et al., 2018). Même constat avec les producteurs d'oignon de la Province de Yatanga au Burkina Faso avec 126 F cfa $/ \mathrm{m}^{2}$ (Napo, 2013). Par contre, les producteurs d'oignon El Soucoucoutane enregistrent des marges nettes plus intéressantes que ceux de Gotheye (en moyenne $412708 \mathrm{~F} \mathrm{cfa} / \mathrm{ha}$ ou $41,27 \mathrm{~F} \mathrm{cfa} / \mathrm{m}^{2}$ ) et d'Ayorou ( $740375 \mathrm{~F} \mathrm{cfa} / \mathrm{ha}$ ou $74,04 \mathrm{~F} \mathrm{cfa} / \mathrm{m}^{2}$ ) [Maman Moustapha et al., 2018]. Il s'avère ainsi, que la culture d'oignon El Soucoucoutane présente des résultats relativement intéressants en termes de revenu pour les producteurs. 
Selon les déclarations des personnes enquêtées, ces revenus constituent une ressource économique inestimable au niveau de la population. Ils servent à l'achat des produits vivriers, aux soins de santé, à l'habillement, à la scolarisation des enfants et aux cérémonies (mariage et baptême). Pour d'autres, ils sont investis dans l'achat d'animaux destinés à l'élevage etc. De l'avis des responsables communaux, la contribution de la filière oignon El Soucoucoutane au budget de la collectivité n'est pas évaluée car il n'y a pas de prélèvement (taxe ou impôt) en dehors des taxes de marché de $100 \mathrm{~F}$ cfa par sac d'oignon.

\section{Conclusion}

La présente étude a relevé que la chaîne de valeurs de l'oignon El Soucoucoutane est une chaîne qu'on peut qualifier de courte, et non structurée. Cette chaîne est à son stade primaire. Il ressort de l'étude une fragilité des maillons de ladite chaîne de valeurs. Pour le maillon de la production, en plus des contraintes liées aux aléas climatiques, l'étude a révélé que l'eau constitue le principal facteur limitant aussi bien en termes de disponibilité que d'accès à la ressource pour l'irrigation. Le circuit de commercialisation est caractérisé notamment par l'absence des commerçants grossistes et la forte saisonnalité du produit sur le marché. C'est ce qui explique en partie le fait que cette variété reste méconnue du grand public en dehors de son bassin de production et alentour.

Cependant, la production d'oignon blanc El Soucoucoutane génère un revenu assez intéressant aussi bien pour les producteurs que pour les commerçants. L'étude a montré que la vente différée d'oignon stocké et bien conservé permet d'avoir une marge bénéficiaire conséquente. Ce produit occupe une place stratégique dans la vie socio-économique des ménages de la zone. En outre, l'étude a mis en évidence l'engagement des producteurs pour la préservation de cette variété à travers leur refus systématique à produire des variétés d'oignon autres qu'El Soucoucoutane. A cela s'ajoute l'option affichée de ces producteurs pour une culture strictement biologique afin de garantir une meilleure conservation du produit et pour conquérir des nouveaux marchés au niveau national et sous-régional. L'enquête a permis de recenser plusieurs vertus médicinales auxquelles la population attache beaucoup d'intérêt. Cet aspect, mérite d'être approfondi pour ajouter de la valeur à la filière oignon blanc de Soucoucoutane.

Dans le cadre de l'amélioration de la compétitivité de la Chaîne de Valeurs de commercialisation d'oignon El Soucoucoutane sur les marchés nationaux voire internationaux, il va falloir travailler davantage sur la promotion de ladite variété, la spécialisation des marchés et le renforcement de la qualité du produit (production et conservation). Aussi, la compétitivité d'une Chaîne de valeurs agricole dépend-elle de la solidité des relations ou 
capacité des acteurs à nouer des relations stratégiques de production ou de commercialisation. Il s'agit là d'un aspect à prendre en compte dans les actions futures car jusque-là la commercialisation d'oignon El Soucoucoutane reste très informelle, individuelle et non structurée.

\section{Remerciements}

Nous remercions l'ONG internationale SWISSAID pour avoir financé les activités de terrain de la présente étude.

\section{References:}

1. Abdou, R., Malice, M., Bakasso, Y., Saadou, M. \& Baudoin, J.P. (2014). Taxonomie locale et analyse des critères des paysans pour caractériser les différents écotypes d'oignons (Allium cepa L.) du Niger. Cahier d'Agriculture, 23(3): pp. 166-76.

2. Assoumane, M. \& Hilali, A. (sd). L'amélioration de l'oignon (Allium cepa L.) au Niger, RADHORT-Documents FAO. [En ligne]: http://www.fao.org/3/a-az835f.pdf (20 Octobre, 2018).

3. Bockel, L. \& Tallec, F. (2005). L'approche filière. Analyse fonctionnelle et identification des flux. Outils analytiques. Module EASYPol043. Rome: FAO, 2005. [En ligne]: http://www.fao.org/docs/up/easypol/417/value_chain_analysis_flow_ charts_043FR.pdf (15 avril 2018).

4. CNRA (2013). Appel à proposition de projets de recherche, 1 ère édition 2013. Amélioration de la production de l'oignon au Niger. Cabinet du premier Ministre, Niamey. Niger. [En ligne]: http://www.recaniger.org/IMG/pdf/OIGNONSvcf2.pdf (10 octobre 2018).

5. CRA \& FCMN (2018). Fiche technico-économique pour la culture d'oignon blanc. Région de Dosso. Niger. 4 p.

6. Currah, L. (2002). Onions in the Tropics: Cultivars and Country Reports, In: H.D. Rabinowitch and L. Currah (Ed.), Allium Crop Science: Recent advances (pp. 379-408). Wallingford (Oxon, UK); New York: CABI Publishing. pp. 379-408.

7. D'Alessandro, S. \& Soumah, A. (2008). Évaluation sous régionale de la chaîne de valeurs oignon/échalote en Afrique de l'Ouest. Bethesda (MD, USA) : projet ATP, Abt Associates Inc. 58 p.

8. DRARHASA (Direction Régionale de l'Agriculture, des Ressources Hydrauliques, de l'Assainissement et de la Sécurité Alimentaire). (sd). Fiche Technique de l'oignon. Ministère de l'Agriculture, des Ressources Hydrauliques, de l'Assainissement et de la Sécurité Alimentaire. Ouaga. Burkina Faso. 7 p. [En ligne]: http://labos.ulg.ac.be/agricotech/wp- 
content/uploads/sites/5/2015/09/Fiche-technique-sur-loignon-R.docx (20 octobre, 2018).

9. Fabre, P. (1994). Note de méthodologie générale sur l'analyse de filière. Utilisation de la filière pour l'analyse économique des politiques. Rome: FAO. (Documents de formation pour la planification agricole; no 35), $105 \mathrm{p}$.

10. INS (2018). Annuaire statistique régional de Dosso. Direction régionale de l'Institut National de la statistique de Dosso. Niger. 153 p.

11. INS (2012). Présentation des résultats globaux définitifs du Quatrième (4ème) Recensement Général de la Population et de l'Habitat $(R G P / H)$ de 2012. Niamey. Niger. 351 p.

12. Lebailly, $\mathrm{Ph}$. (1990). Concept de filière, économie agro-alimentaire et développement. Tropicultura. 8(1) : pp. 9-14.

13. Maman Moustapha, R., Idrissa, M., Tchicama Mella, M. \& Hassimi, M. (2018). Panorama of Onion Production in Tillabéri, A Region of the Far West of Niger. European Scientific Journal. 14 (15): pp.175196.

[En

ligne] :

http://eujournal.org/index.php/esj/article/view/10867/10401

$(20$ octobre 2018).

14. Nabos, J. (1976). L'amélioration de l'oignon (Allium cepa L.) au Niger. Agronomie Tropicale (XXXI) : pp. 387-97.

15. Napo, H.I. (2013). Etude diagnostique des techniques de production de l'oignon (Allium cepa L.) dans la province du Yatenga. Mémoire de Master. Université Polytechnique de Bobo-dioulasso (U.P.B), Institut du Développement Rural (I.D.R), Burkina Faso, [Enligne] : http://www.beep.ird.fr/collect/upb/index/assoc/IDR-2013NAPETU/IDR-2013-NAP-ETU.pdf. (20 octobre 2018). 53 p.

16. Phan Dang, Th. (2013). La filière avicole de chair dans les zones périurbaines de Hanoï, Vietnam: situation économique et perspectives (Thèse de doctorat). Université de Liège - Gembloux Agro-Bio Tech, Belgique, $183 \mathrm{p}$.

17. PRODEX (2013). Guide d'exportation de l'oignon. Ministère de l'Agriculture. Niamey. Niger. $24 \mathrm{p}$.

18. PRODEX (2012). Guide de bonnes pratiques de production, stockage et conservation de l'oignon, $1^{\text {ère }}$ édition. Ministère de l'Agriculture. Niamey, Niger.

19. RECA (2011). L'importance de l'oignon dans la vie socioéconomique du Niger : contexte et agendas d'action. Note d'information / Filière oignon $n^{\circ} 8$, Niamey. Niger, $4 \mathrm{p}$. 
20. RECA (Réseau National des Chambres d'Agriculture du Niger). (2010). Combien d'oignons sont produits au Niger...c'est (très) difficile à savoir ! In RECA Info, no. 8, Page 3 et pp 10-11.

21. Rouamba, A., Ricroch A., \& Sarr, A. (1997). Dynamic management of genetic resources of Allium cepa L. (onion) in West Africa. In Acta Horticulturae ; doi: 10.17660/ ActaHortic.1997.433.18. (433, pp. 185190).

22. Seck, A. (2014). Fiche technique de production intensive de l'oignon. Programme d'Aménagement et de Développement Economique des Niayes (PADEN). Ministère de l'Agriculture et de l'équipement rural, Sénégal. (47 pages).

23. Tarchiani, V., Robbiati, G., \& Salifou, M.R. (2013). Filières oignon en Afrique de l'Ouest: étude comparée des filières nigérienne et béninoise. Cahier d'Agriculture, 22 (2): pp. 112-23. doi : 23.1684/agr.2013.0617, [En ligne] :

http://revues.cirad.fr/index.php/cahiersagricultures/article/view/31017 (le 31 octobre 2018).

24. Van Vugt, S.M., Schrader, T.H., De Roo, N., Daddy, A., Douma, A., \& Salifou, M.L. (2010). L'importance de l'oignon dans la vie socioéconomique du Niger. Contexte et agenda d'action, Rapport de l'atelier «Eplucher l'oignon». Birni N'Konni, Niger, novembredécembre 2010. 58 p. 